\section{CardioRenal Medicine}

\title{
Epicardial Fat Thickness in Patients with Autosomal Dominant Polycystic Kidney Disease
}

\author{
Antonio Concistrèa Luigi Petramala ${ }^{a}$ Gianmarco Scoccia ${ }^{a}$ \\ Susanna Sciomer ${ }^{b}$ Valeria Bisogni ${ }^{a}$ Vincenza Saracino ${ }^{a}$ Gino lannucci $^{a}$ \\ Silvia Lai ${ }^{c}$ Daniela Mastroluca ${ }^{d}$ Gianluca lacobellis $^{\mathrm{e}}$ Claudio Letizia $^{a}$ \\ aUnit of Secondary Hypertension, Department of Internal Medicine and Medical Specialties, \\ Sapienza University of Rome, Rome, Italy; ${ }^{b}$ Department of Cardiovascular and Respiratory \\ Sciences, Sapienza University of Rome, Rome, Italy; ' Department of Clinical Medicine, \\ Sapienza University of Rome, Rome, Italy; ${ }^{d}$ Nephrology and Dialysis Unit, Hospital ICOT

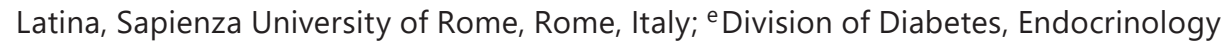 \\ and Metabolism, Department of Medicine, University of Miami, Miller School of Medicine, \\ Miami, FL, USA
}

\section{Keywords}

Epicardial fat thickness - Autosomal dominant polycystic kidney disease $\cdot$ Cardiovascular disease $\cdot$ Left ventricular hypertrophy

\begin{abstract}
Introduction: Autosomal dominant polycystic kidney disease (ADPKD) is associated with early organ damage such as left ventricular hypertrophy and higher cardiovascular risk when compared to essential hypertension (EH). Epicardial adipose tissue (EAT) is a new cardiovascular risk factor, but its role and correlation with left ventricular mass (LVM) in ADPKD is unknown. Aims: we sought to investigate whether EAT is higher and related to LVM indexed by body surface area (LVMi) in hypertensive patients with ADPKD compared to those with EH. Methods: We performed ultrasound measurement of EAT thickness, LVM, LVMi, and left atrium size (left atrial volume indexed for body surface, LAVI) in 41 consecutive hypertensive patients with ADPKD, compared to 89 EH patients. Results: EAT was significantly higher in the ADPKD group in comparison to EH subjects $(9.2 \pm 2.9 \mathrm{~mm}$ vs. $7.8 \pm 1.6 \mathrm{~mm}, p<0.001)$, and significantly correlated with LVM, LVMi, and LAVI in the ADPKD group $(r=0.56, p=0.005 ; r=$ $0.424, p=0.022$; and $r=0.48, p=<0.001$, respectively). Comparing EAT against body mass index, systolic blood pressure, and age, we found that EAT was the strongest predictor of LVMi ( $\beta=0.42, p=0.007$ ). Conclusion: Our data showed that EAT was higher in ADPKD patients than in EH subjects and independently correlated with LVMi. EAT measurement can be a useful marker for the cardiovascular risk stratification in ADPKD.




\section{Introduction}

Autosomal dominant polycystic kidney disease (ADPKD) is the most common genetic kidney disease, characterized by the formation of cystic dilatation of renal tubules with progressive destruction of renal parenchyma continuing to an early onset of end-stage renal disease [1-3]. ADPKD patients are at higher cardiovascular risk, and cardiovascular death is estimated to be 1.6 - to 3.2 -fold higher in these patients than in the general population $[4,5]$. Hypertension is commonly associated with ADPKD, occurring in 50-70\% of patients even before the progression to renal dysfunction [6]. Left ventricular hypertrophy (LVH), a major cardiovascular risk factor, is also frequently observed in patients with ADPKD [7, 8]. Both hypertension and LVH play a crucial role in the development of cardiovascular complications in these patients. Traditional risk factors, such as blood pressure (BP) control, proteinuria, body mass index (BMI), age, renal function, and insulin resistance have all been independently associated with left ventricular mass (LVM) and LVM indexed by body surface area (LVMi) in ADPKD [9-11]. Obesity has recently and independently been associated with progression in early-stage ADPKD [12]. Nevertheless, whether visceral obesity and specifically the adiposity of the heart, namely the epicardial adipose tissue (EAT), can play a role in ADPKD and in the development of LVH in these patients is unexplored. EAT is thought to directly and independently modulate the heart, due to its proximity to the coronary arteries and paracrine interaction with the myocardium $[13,14]$. EAT has been shown to correlate with LVMi in conditions of insulin resistance, such as obesity and diabetes [15]. EAT thickness can be easily visualized and measured using standard 2D echocardiography, as first developed and validated by Iacobellis and Willens [16].Nowadays, the relationship between EAT and LVMi in hypertensive ADPKD patients is unknown. Hence, in this study we sought to investigate whether EAT could be higher and related to LVMi in hypertensive patients with ADPKD compared to those with essential hypertension (EH).

\section{Patients and Methods}

From January 2015 to April 2016, we enrolled 41 consecutive patients (20 men, 21 women, mean age $44 \pm 15$ years, age range 19-78 years), who were diagnosed with ADPKD in the Nephrology Unit, Sapienza University of Rome, Italy, and were referred to the Tertiary Centre of Secondary Hypertension Unit, Sapienza University of Rome. Eighty-nine patients with EH were recruited as the control group (mean age $49 \pm 12$ years, age range 17-82 years). Anthropometric measurements and fasting venous blood samples were obtained from all patients. Clinical BP was measured during the visit according to guidelines [17], and the diagnosis of hypertension was made if systolic BP (SBP) was found to be $\geq 140 \mathrm{~mm} \mathrm{Hg}$ and diastolic BP (DBP) $\geq 90 \mathrm{~mm} \mathrm{Hg}$ [17] for three consecutive measurements with the sphygmomanometer by the same investigator. Patients with clinical history or clinical symptoms or electrocardiogram, echocardiographic, and angiographic signs of coronary artery disease, heart failure, cardiomyopathies, or valvular or pericardial diseases were excluded. Patients with cerebrovascular and peripheral artery diseases or hepatic diseases, and patients with a history of cardiovascular or cerebrovascular events, smoking, and drug abuse were excluded. Patients with an established diagnosis of type 2 diabetes were also not included in this study to avoid the possible confounding effects of diabetes on study results. Renal disease was defined as the presence of serum creatinine $>1.5 \mathrm{mg} / \mathrm{dL}$, estimated glomerular filtration rate (eGFR) $<60 \mathrm{~mL} / \mathrm{min}$, or albuminuria $>300 \mathrm{mg} / 24 \mathrm{~h}$. All the study participants followed a regular dietary habit with a salt intake of $140 \mathrm{mEq} /$ day and potassium intake of 50-75 mEq/day. All patients had stable body weight for 6 months before the study.

Diagnosis of EH

EH was defined as BP of 140/90 mm Hg or more in three consecutive measurements (SBP and DBP). Secondary causes of hypertension were all excluded on the basis of clinical, laboratory, and imaging examinations. 
Concistrè et al.: Epicardial Fat Thickness in ADPKD

Table 1. Anthropometric and clinical parameters in patients with autosomal polycystic kidney disease (ADPKD) and essential hypertension (EH)

\begin{tabular}{lccc}
\hline & ADPKD $(n=41)$ & EH $(n=89)$ & $p$ \\
\hline Age, years & $44 \pm 15$ & $49 \pm 12$ & $\mathrm{~ns}$ \\
Sex (M/F), $n$ & $21: 20$ & $49: 40$ & $\mathrm{~ns}$ \\
BMI & $26 \pm 4$ & $26 \pm 3$ & $\mathrm{~ns}$ \\
WC, cm & $92.6 \pm 13$ & $94 \pm 15$ & $\mathrm{~ns}$ \\
SBP, mm Hg & $141 \pm 11$ & $142 \pm 15$ & $\mathrm{~ns}$ \\
DBP, mm Hg & $87 \pm 9$ & $88 \pm 10$ & $\mathrm{~ns}$ \\
Creatinine, mg/dL & $1.2 \pm 0.18$ & $0.9 \pm 2$ & $\mathrm{~ns}$ \\
eGFR, mL/min/1.73 m ${ }^{2}$ & $75 \pm 15$ & $83 \pm 19$ & $\mathrm{~ns}$ \\
FG, mg/dL & $92 \pm 11$ & $90 \pm 15$ & $\mathrm{~ns}$ \\
Na ${ }^{+}, \mathrm{mEq} / \mathrm{L}$ & $141 \pm 3$ & $142 \pm 2$ & $\mathrm{~ns}$ \\
$\mathrm{~K}^{+}, \mathrm{mEq} / \mathrm{L}$ & $4.5 \pm 0.4$ & $4.2 \pm 0.4$ & $\mathrm{~ns}$ \\
Uric acid, mg/dL & $5.6 \pm 1.3$ & $5.3 \pm 1.5$ & $\mathrm{~ns}$ \\
TC, mg/dL & $183.8 \pm 27.7$ & $200.51 \pm 37.98$ & $\mathrm{~ns}$ \\
LDL-C, mg/dL & $109.9 \pm 25.8$ & $120.7 \pm 31.5$ & $\mathrm{~ns}$ \\
HDL-C, mg/dL & $55.9 \pm 15.2$ & $55.9 \pm 15.4$ & $\mathrm{~ns}$ \\
TGL, mg/dL & $95.6 \pm 44.0$ & $99.2 \pm 48.9$ & \\
\hline
\end{tabular}

Data are expressed as means \pm standard deviations unless otherwise indicated. BMI, body mass index; WC, waist circumference; SBP, systolic blood pressure; DBP, diastolic blood pressure; FG, fasting blood glucose; $\mathrm{Na}^{+}$, sodium; $\mathrm{K}^{+}$, potassium; TC, total cholesterol; LDL-C, low-density lipoprotein cholesterol; HDL-C, high-density lipoprotein cholesterol; TGL, triglycerides; ns, not significant.

Epicardial Fat Thickness, LVM, and Diastolic Dysfunction

Each subject had a transthoracic 2D-guided M-mode echocardiogram using commercially available equipment (Siemens Sonoline G20). Standard parasternal and apical views were obtained with patients lying in the left lateral decubitus position. All echocardiograms were recorded and analyzed offline for EAT thickness quantification, according to the methodology previously described and validated by Iacobellis et al. $[15,16]$. EAT was identified as the echo-free space between the outer wall of the myocardium and the visceral layer of the pericardium. EAT thickness was measured perpendicularly on the free wall of the right ventricle at end-systole in three cardiac cycles. Maximum EAT thickness was measured at the point on the free wall of the right ventricle along the midline of the ultrasound beam, perpendicular to the aortic annulus, which was used as an anatomic landmark for this view. The average value of three cardiac cycles from each echocardiographic view was considered. LVM and LVMi were calculated as previously described [18]. LVH was defined as LVMi of $115 \mathrm{~g} / \mathrm{m}^{2}$ or more for men and $95 \mathrm{~g} / \mathrm{m}^{2}$ or more for women [19]; diastolic dysfunction was defined according to the American Society of Echocardiography (ASE) guidelines [20].

\section{Statistical Analysis}

All data are expressed as mean \pm standard deviation $( \pm \mathrm{SD})$. Differences between means were assessed by the Student $t$ test or the Mann-Whitney U test in nonnormally distributed data for two-sample comparison, or by one-way analysis of variance (ANOVA) applying the Fisher least significant difference post hoc test for multiple comparisons. $\chi^{2}$ statistics were used to assess differences between categorical variables. Relationships between continuous variables were assessed calculating the Pearson correlation coefficient or the Spearman rank correlation coefficient when appropriate. Multiple linear regression analysis with the "Enter" method was used to identify the statistically significant predicting factors of LVMi. A multivariate logistic regression model was used to identify independent predictors of LVMi. Both of the analyses stated above were undertaken in the entire cohort of patients. We compared the predictive performance of EAT and LVMi as continuous variables using receiver operating characteristic (ROC) curves and by calculating the area under the curves. $p$ values < 0.05 were taken as statistically significant. Statistical analysis and ROC curve analysis was performed using the Statistical Package for the Social Sciences (SPSS-PC version 24.0; SPSS Inc., Chicago, IL, USA). 
Concistrè et al.: Epicardial Fat Thickness in ADPKD

Table 2. Echocardiographic parameters in patients with autosomal polycystic kidney disease (ADPKD) and essential hypertension (EH)

\begin{tabular}{lccc}
\hline & ADPKD $(n=41)$ & $\mathrm{EH}(n=89)$ & $p$ \\
\hline EAT, mm & $9.2 \pm 2.9$ & $7.8 \pm 1.6$ & $<0.001$ \\
IVSd, mm & $10 \pm 1.6$ & $8.3 \pm 1.7$ & $<0.001$ \\
LVPWd, mm & $9.2 \pm 1.5$ & $8.3 \pm 1.6$ & $<0.001$ \\
LAVI, mL/m ${ }^{2}$ & $24 \pm 3.9$ & $20.7 \pm 4.1$ & $<0.001$ \\
LVM, g & $181.87 \pm 54.9$ & $125.21 \pm 25.7$ & $<0.001$ \\
LVMi, g/m ${ }^{2}$ & $98.9 \pm 31.5$ & $71.9 \pm 12.5$ & $<0.001$ \\
LVEF, \% & $61.6 \pm 4.6$ & $61.3 \pm 4.3$ & $\mathrm{~ns}$ \\
Diastolic dysfunction grade I, $n(\%)$ & $18(43)$ & $44(49)$ & $\mathrm{ns}$ \\
LVH, $n(\%)$ & $9(22)$ & 0 & 0.001 \\
\hline
\end{tabular}

Data are expressed as means \pm standard deviations unless otherwise indicated. EAT, epicardial adipose tissue; IVSd, end-diastolic interventricular septum; LVPWd, end-diastolic left ventricular posterior wall; LAVI, left atrium volume indexed for body surface area; LVM, left ventricle mass; LVMI, left ventricle mass indexed for body surface area; LVH, left ventricular hypertrophy.

Table 3. Multiple regression analysis comparing EAT against age, BMI, SBP, DBP, and WC in predicting LVMi

\begin{tabular}{lllll}
\hline & $\beta$ & $\begin{array}{l}95 \% \mathrm{CI} \\
\text { inferior limit }\end{array}$ & $\begin{array}{l}\text { 95\% CI } \\
\text { superior limit }\end{array}$ & $p$ \\
\hline EAT & 0.417 & 1.220 & 7.299 & 0.007 \\
Age & -0.076 & -0.749 & 0.436 & 0.596 \\
BMI & 0.178 & -1.057 & 2.685 & 0.385 \\
SBP & 0.033 & -0.435 & 0.547 & 0.819 \\
\hline
\end{tabular}

The regression model was obtained after the exclusion of the variables DBP and WC because of a high variance inflation factor which indicates a high level of collinearity; after excluding the collinearity we achieved a regression model with $R^{2}=0.61$. EAT, epicardial adipose tissue; BMI, body mass index; SBP, systolic blood pressure; DBP, diastolic blood pressure; WC, waist circumference; LVMi, left ventricular mass indexed by body surface area.

\section{Results}

Characteristics of the study patients are summarized in Table 1. The two groups (ADPKD and $\mathrm{EH}$ ) did not differ by age, sex distribution, BMI, waist circumference (WC), or BP values.

EAT was significantly higher in the ADPKD group compared to the EH group $(9.2 \pm 2.9$ vs. $7.8 \pm 1.6 \mathrm{~mm}, p<0.001$ ), as reported in Table 2. End-diastolic interventricular septum (IVSd), left ventricular posterior wall (LVPWd), left atrial volume indexed for body surface (LAVI), LVM, and LVMi measurements were also higher in the ADPKD group compared to controls $(p<0.001)$. None of the patients with EH had LVH, whereas 9 patients affected by ADPKD presented LVH $(p=0.001)$. No differences in the diastolic parameters were found between the groups (Table 2). In a linear regression model EAT was significantly correlated with LVM and LVMi in the ADPKD group ( $r=0.56, p=0.005$ and $r=0.424, p=0.022$, respectively). EAT correlated better with both LVM and LVMi than with BMI $(r=-0.19, p=0.25)$ and WC $(r=$ $-0.21, p=0.35)$. No correlation was found between EAT and LVM/LVMi in the EH group $(r=$ $0.08, p=0.61$ and $r=0.003, p=0.98$, respectively). An additional positive correlation was observed between EAT and left atrium size $(r=0.48 ; p=<0.001)$. In a multiple regression 


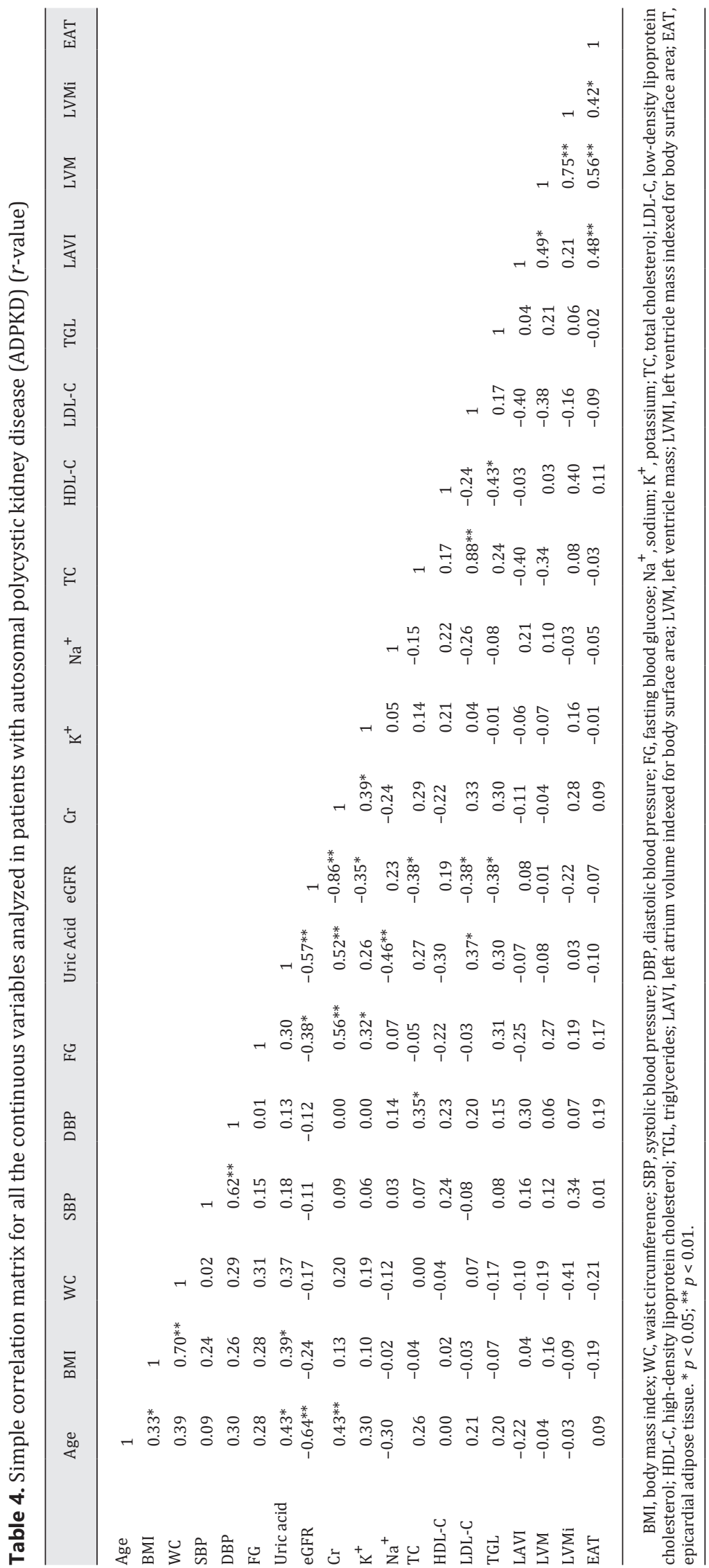




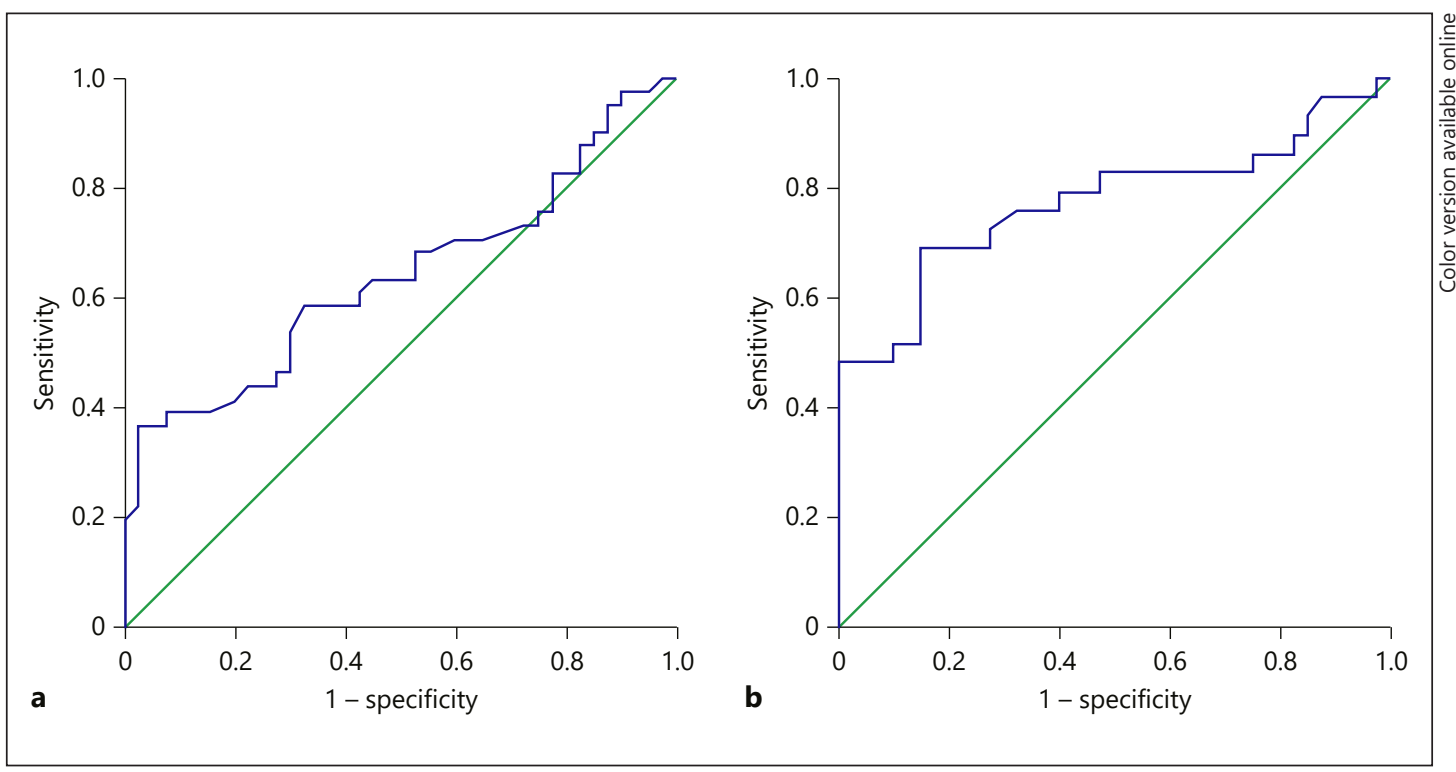

Fig. 1. ROC curve analysis compares the diagnostic performance of epicardial fat thickness (EAT; a) and left ventricular mass indexed by body surface area (LVMi; b) to discriminate autosomal dominant polycystic kidney disease. $\mathrm{AUC}$ for $\mathrm{EAT}=0.639$; $\mathrm{AUC}$ for $\mathrm{LVMi}=0.774$.

analysis model including LVMi as dependent variable and EAT, BMI, and WC as independent variables, EAT was the only statistically significant correlate of LVMi ( $\beta=0.59, p=0.036$ vs. $\beta=0.431, p=0.347$ for BMI and $\beta=-0.563, p=0.225$ for WC). Additional multiple regression analysis comparing EAT against BMI, SBP, and age showed EAT as the only parameter correlating with LVMi (Table 3). Table 4 shows the simple correlation matrix for the continuous variables analyzed. In Figure 1 we report the ROC curve analysis comparing the diagnostic performance of EAT and LVMi to discriminate ADPKD.

\section{Discussion}

Cardiovascular complications, including arterial hypertension and myocardial and valvular diseases, represent the major cause of morbidity and mortality in patients with ADPKD [4, 7, 21-24]. In this study we first demonstrated that (1) EAT was higher in patients with ADPKD and hypertension and normal renal function compared to patients with EH and (2) EAT was the strongest correlate of LVMi in patients with ADPKD irrespective of other traditional risk factors such as BMI, WC, and BP. EAT is a novel cardiometabolic risk factor that has been widely linked to LVH, subclinical atherosclerosis, and coronary artery disease $[25,26]$. Data on EAT in patients with ADPKD are quite scarce and its relation with LVH was not explored before. However, ultrasound-measured EAT thickness was recently associated with carotid intima-media thickness, a well-accepted marker of subclinical atherosclerosis, in patients with ADPKD [27].

In light of the recently suggested independent role of obesity in the early development of ADPKD, we believe that our findings are of particular interest and novelty [12]. Obesity is a well-established independent risk factor for incident CKD, end-stage renal disease, and a decline in eGFR in the general population. The proinflammatory status commonly described in obesity is considered one of the factors implicated in the development of CKD and also 
ADPKD, as recently suggested [12]. Remarkably, EAT is a highly inflammatory fat depot that plays a key pathogenic role in conditions that are not necessarily linked to obesity, such as coronary artery disease and CKD [28]. EAT has a unique proteasome and transcriptome highly enriched in genes encoding for inflammatory factors, as recently described by Iacobellis and colleagues [29]. Ultrasound-measured EAT thickness showed a correlation with circulating inflammatory markers $[13,14,18]$. Hence, based on the proinflammatory properties of EAT and the recently suggested role of obesity-related inflammation in ADPKD, it was somehow anticipated that EAT was higher in these subjects than in those with EH. Future studies exploring EAT inflammatory proteasome and transcriptome in subjects with ADPKD seem to be warranted.

ADPK is commonly associated with increased LVMi and LVH. The development of LVH in patients with ADPKD is likely multifactorial and includes activation of the renin-angiotensinaldosterone system, increase of sympathetic nervous system activity, endothelial dysfunction, and left ventricular diastolic dysfunction [30]. Our study suggests that EAT can also play a pathogenic role in ADPKD-related LVH. In particular, we found a strong and independent correlation between EAT and LVMi, although ROC curve analysis showed a superiority of LVMi in predicting cardiovascular risk in ADPKD subjects. Although, no conclusion on the cause-effect of the EAT-LVM relation can be drawn from our study, some speculations on the mechanisms can be mentioned. EAT is thought to locally affect the heart throughout the paracrine or vasocrine secretion of proinflammatory and profibrotic cytokines. Other mechanical and biomolecular mechanisms could be also evoked to explain the effect of EAT on the heart. Increased EAT by adding mass to both ventricles increases the work of the heart and then leads to LVH. An infiltration of adipocytes from EAT to the myocardium has also been suggested [31].

Finally, we found and confirmed that the patients with ADPKD and arterial hypertension had higher LVMi left atrium size, IVSd, and LVPWd than EH patients [32]. The correlation between EAT and the left atrium was never detected before in ADPKD patients.

This finding could be of particular interest in light of the growing association between EAT and atrial fibrillation. Mechanical and biochemical factors have been suggested to play a role in this association, although the mechanisms are still unclear [33-35].

EAT thickness can be easily and accurately measured with standard ultrasound in a hypertension work-up, as previously shown by our group [36]. We believe that LVMi and EAT are not interchangeable. They provide two different sets of information that can complement each other. It is an advantage that both can be not invasively obtained. Although this study did not aim to evaluate the superiority of one measurable risk factor over another, EAT could also be a useful diagnostic tool for stratification of the cardiometabolic risk in patients with ADPKD [37].

\section{Conclusions}

This study provides novel findings that may have a clinical application for cardiovascular risk assessment in patients with ADPKD. Our data show that EAT is higher in patients with ADPKD than in EH patients with the same pressure load. Therefore, patients with ADPKD could benefit from an early evaluation of EAT.

\section{Study Limitations}

No conclusions on cause-effect mechanisms can be drawn from this study. Although our sample size had the power to detect statistically significant differences between the groups, larger and longitudinal studies are warranted. The use of antihypertensive medications could 
influence the study results. Global longitudinal strain was not available and therefore could not allow a more accurate assessment of the left ventricular function. To address the potential issue of an operator-dependent procedure, all EAT measurements were performed by the same operator utilizing the same technique.

\section{Statement of Ethics}

The study was conducted in accordance with the Declaration of Helsinki. Informed consent was obtained from all individual participants included in the study.

\section{Disclosure Statement}

The authors declare that there is no conflict of interest that could be perceived as prejudicing the impartiality of the research reported. This research did not receive any specific grant from any funding agency in the public, commercial, or nonprofit sector.

\section{References}

1 Grantham JJ: Clinical practice. Autosomal dominant polycystic kidney disease. N Engl J Med 2008;359:14771485.

-2 Iglesias CG, Torres VE, Offord KP, Holley KE, Beard CM, Kurland LT: Epidemiology of adult polycystic kidney disease, Olmsted County, Minnesota: 1935-1980. Am J Kidney Dis 1983;2:630-639.

-3 Igarashi P, Somlo S: Genetics and pathogenesis of polycystic kidney disease. J Am Soc Nephrol 2002;13:23842398.

-4 Luciano RL, Dahl NK: Extra-renal manifestations of autosomal dominant polycystic kidney disease (ADPKD): considerations for routine screening and management. Nephrol Dial Transplant 2014;29:247-254.

-5 Florijn KW, Noteboom WM, van Saase JL, Chang PC, Breuning MH, Vandenbroucke JP: A century of mortality in five large families with polycystic kidney disease. Am J Kidney Dis 1995;25:370-374.

-6 Chapman AB, Stepniakowski K, Rahbari-Oskoui F: Hypertension in autosomal dominant polycystic kidney disease. Adv Chronic Kidney Dis 2010;17:153-163.

7 Ecder T, Schrier RW: Cardiovascular abnormalities in autosomal-dominant polycystic kidney disease. Nat Rev Nephrol 2009;5:221-228.

8 Martinez-Vea A, Valero FA, Bardaji A, Gutierrez C, Broch M, Garcia C, Richart C, Oliver JA: Left ventricular hypertrophy in hypertensive patients with autosomal dominant polycystic kidney disease: influence of blood pressure and humoral and neurohormonal factors. Am J Nephrol 2000;20:193-200.

-9 Bardají A, Martinez-Vea A, Valero A, Gutierrez C, Garcia C, Ridao C, Oliver JA, Richart C: Cardiac involvement in autosomal-dominant polycystic kidney disease: a hypertensive heart disease. Clin Nephrol 2001;56:211220.

10 Chapman AB, Johnson AM, Rainguet S, Hossack K, Gabow P, Schrier RW: Left ventricular hypertrophy in autosomal dominant polycystic kidney disease. J Am Soc Nephrol 1997;8:1292-1297.

-11 Lumiaho A, Pihlajamäki J, Hartikainen J, Ikäheimo R, Miettinen R, Niemitukia L, Lampainen E, Laakso M: Insulin resistance is related to left ventricular hypertrophy in patients with polycystic kidney disease type 1. Am J Kidney Dis 2003;41:1219-1224.

12 Nowak KL, You Z, Gitomer B, Brosnahan G, Torres VE, Chapman AB, Perrone RD, Steinman TI, Abebe KZ, Rahbari-Oskoui FF, Yu ASL, Harris PC, Bae KT, Hogan M, Miskulin D, Chonchol M: Overweight and obesity are predictors of progression in early autosomal dominant polycystic kidney disease. J Am Soc Nephrol 2018;29: 571-578.

13 Iacobellis G, Bianco AC: Epicardial adipose tissue: emerging physiological, pathophysiological and clinical features. Trends Endocrinol Metab 2011;22:450-457.

14 Iacobellis G: Local and systemic effects of the multifaceted epicardial adipose tissue depot. Nat Rev Endocrinol 2015;11:363-371.

-15 Iacobellis G, Ribaudo MC, Zappaterreno A, Iannucci CV, Leonetti F: Relation between epicardial adipose tissue and left ventricular mass. Am J Cardiol 2004;94:1084-1087.

16 Iacobellis G, Willens HJ: Echocardiographic epicardial fat: a review of research and clinical applications. J Am Soc Echocardiogr 2009;22:1311-1319. 
Mancia G, Fagard R, Narkiewicz K, Redón J, Zanchetti A, Böhm M, Christiaens T, Cifkova R, De Backer G, Dominiczak A, Galderisi M, Grobbee DE, Jaarsma T, Kirchhof P, Kjeldsen SE, Laurent S, Manolis AJ, Nilsson PM, Ruilope LM, Schmieder RE, Sirnes PA, Sleight P, Viigimaa M, Waeber B, Zannad F; Task Force Members: 2013 ESH/ESC Guidelines for the management of arterial hypertension: the Task Force for the management of arterial hypertension of the European Society of Hypertension (ESH) and of the European Society of Cardiology (ESC). J Hypertens 2013;31:1281-1357.

18 Iacobellis G, Ribaudo MC, Assael F, Vecci E, Tiberti C, Zappaterreno A, Di Mario U, Leonetti F: Echocardiographic epicardial adipose tissue is related to anthropometric and clinical parameters of metabolic syndrome: a new indicator of cardiovascular risk. J Clin Endocrinol Metab 2003;88:5163-5168.

19 Lang RM, Bierig M, Devereux RB, Flachskampf FA, Foster E, Pellikka PA, Picard MH, Roman MJ, Seward J, Shanewise JS, Solomon SD, Spencer KT, Sutton MS, Stewart WJ; Chamber Quantification Writing Group; American Society of Echocardiography's Guidelines and Standards Committee; European Association of Echocardiography: Recommendations for chamber quantification: a report from the American Society of Echocardiography's Guidelines and Standards Committee and the Chamber Quantification Writing Group, developed in conjunction with the European Association of Echocardiography, a branch of the European Society of Cardiology. J Am Soc Echocardiogr 2005;18:1440-1463.

20 Nagueh SF, Smiseth OA, Appleton CP, Byrd BF 3rd, Dokainish H, Edvardsen T, Flachskampf FA, Gillebert TC, Klein AL, Lancellotti P, Marino P, Oh JK, Alexandru Popescu B, Waggoner AD: Recommendations for the evaluation of left ventricular diastolic function by echocardiography: an update from the American Society of Echocardiography and the European Association of Cardiovascular Imaging. Eur Heart J Cardiovasc Imaging 2016; 17:1321-1360.

-21 Badani KK, Hemal AK, Menon M: Autosomal dominant polycystic kidney disease and pain - a review of the disease from aetiology, evaluation, past surgical treatment options to current practice. J Postgrad Med 2004; 50:222-226 Ryu SJ: Intracranial hemorrhage in patients with polycystic kidney disease. Stroke 1990;21:291-294. Torres VE, Harris PC, Pirson Y: Autosomal dominant polycystic kidney disease. Lancet 2007;369:1287-1301. Perrone RD, Abebe KZ, Schrier RW, et al: Cardiac magnetic resonance assessment of left ventricular mass in autosomal dominant polycystic kidney disease. Clin J Am Soc Nephrol 2011;6:2508-2515.

25 Pierdomenico SD, Pierdomenico AM, Cuccurullo F, Iacobellis G: Meta-analysis of the relation of echocardiographic epicardial adipose tissue thickness and the metabolic syndrome. Am J Cardiol 2013;111:73-78.

-26 Iacobellis G, Corradi D, Sharma AM: Epicardial adipose tissue: anatomic, biomolecular and clinical relationships with the heart. Nat Clin Pract Cardiovasc Med 2005;2:536-543.

27 Sag S, Yildiz A, Gullulu S, Gungoren F, Ozdemir B, Cegilli E, Oruc A, Ersoy A, Gullulu M: Early atherosclerosis in normotensive patients with autosomal dominant polycystic kidney disease: the relation between epicardial adipose tissue thickness and carotid intima-media thickness. Springerplus 2016;5:211.

-28 Cordeiro AC, Amparo FC, Oliveira MA, Amodeo C, Smanio P, Pinto IM, Lindholm B, Stenvinkel P, Carrero JJ: Epicardial fat accumulation, cardiometabolic profile and cardiovascular events in patients with stages 3-5 chronic kidney disease. J Intern Med 2015;278:77-87.

29 McAninch EA, Fonseca TL, Poggioli R, Panos AL, Salerno TA, Deng Y, Li Y, Bianco AC, Iacobellis G: Epicardial adipose tissue has a unique transcriptome modified in severe coronary artery disease. Obesity (Silver Spring) 2015;23:1267-1278.

30 Ecder T, Schrier RW: Hypertension and left ventricular hypertrophy in autosomal dominant polycystic kidney disease. Expert Rev Cardiovasc Ther 2004;2:369-374.

-31 Malavazos AE, Di Leo G, Secchi F, Lupo EN, Dogliotti G, Coman C, Morricone L, Corsi MM, Sardanelli F, Iacobellis G: Relation of echocardiographic epicardial fat thickness and myocardial fat. Am J Cardiol 2010;105:18311835.

-32 Iacobellis G, Malavazos AE, Corsi MM: Epicardial fat: from the biomolecular aspects to the clinical practice. Int J Biochem Cell Biol 2011;43:1651-1654.

-33 Khawaja T, Greer C, Chokshi A, Chavarria N, Thadani S, Jones M, Schaefle K, Bhatia K, Collado JE, Shimbo D, Einstein AJ, Schulze PC: Epicardial fat volume in patients with left ventricular systolic dysfunction. Am J Cardiol 2011;108:397-401.

-34 Lin YK, Chen YJ, Chen SA: Potential atrial arrhythmogenicity of adipocytes: implications for the genesis of atrial fibrillation. Med Hypotheses 2010;74:1026-1029.

35 Iacobellis G, Zaki MC, Garcia D, Willens HJ: Epicardial fat in atrial fibrillation and heart failure. Horm Metab Res 2014;46:587-590.

-36 Iacobellis G, Petramala L, Marinelli C, Calvieri C, Zinnamosca L, Concistrè A, Iannucci G, De Toma G, Letizia C: Epicardial fat thickness and primary aldosteronism. Horm Metab Res 2016;48:238-241.

-37 Iacobellis G, Willens HJ, Barbaro G, Sharma AM: Threshold values of high-risk echocardiographic epicardial fat thickness. Obesity (Silver Spring) 2008;16:887-892. 